\title{
Corneal Endothelium Image Segmentation Using Feedforward Neural Network
}

\author{
Anna Fabijańska \\ Institute of Applied Computer Science \\ Lodz University of Technology \\ ul. Stefanowskiego 18/22, \\ 90-924 Lodz, Poland \\ Email: anna.fabijanska@p.lodz.pl
}

\begin{abstract}
In this paper the problem of corneal endothelium image segmentation is considered. Particularly, a fully automatic approach for delineating contours of corneal endothelial cells is proposed. The approach produces one pixel width outline of cells. It bases on a simple feedforward neural network trained to recognize pixels which belong to the cell borders. The edge probability (edginess) map output by the network is next analysed row by row and column by column in order to find local peaks of the network response. These peaks are considered as cell border candidates and in the last step of the method via binary morphological processing are linked to create continuous outlines of cells. The results of applying the proposed approach to publicity available data set of corneal endothelium images as well as the assessment of the method against ground truth segmentation are presented and discussed. Obtained results show, that the proposed approach performs very well. The resulting mean absolute error of cell number determination is around $5 \%$ while the average DICE measure reaches 0.83 which is a good result, especially when one pixel width objects are compared.

Index Terms - corneal endothelium, cell segmentation, feedfor-
\end{abstract} ward neural network, peaks detection

\section{INTRODUCTION}

$\mathbf{T}$ HE corneal endothelium i.e. the inner layer of the cornea, is of great interest for ophthalmologists. This layer is formed by closely packed, predominantly hexagonal cells whose shape and structure can provide important diagnostic information about the cornea health status or indicate some corneal diseases [1], [2]. Particularly, the quantification of corneal health status is usually performed based on endothelial image by means of corneal endothelial cell density. Additional measures like cell size distribution or cells hexagonality are also useful to evaluate the health status of the corneal tissue. However, the usage of the latter measures is not common in everyday clinical routine. It is because performing this kind of assessment requires segmentation of all cells present in the endothelial image. Having in mind that in the healthy cornea there are up to 3000 endothelial cells per square millimetre, their manual segmentation is very tedious and very time consuming activity. The reason is that it requires manual delineation of cell borders. Since no commercial software is available for corneal endothelial cell segmentation, the development of the dedicated image processing and analysis algorithms for computer aided diagnosis of corneal diseases still remains a vital problem [3].
Segmentation of corneal endothelial cells is a difficult and sometimes very challenging task. The problems arise mainly due to inhomogeneous background illumination in specular microscopy corneal endothelium images. This factor reduces contrast in some regions of an image and makes cell borders difficult to recognize even by an expert.

Several semi-automatic or fully automatic solutions for segmentation of endothelial image have already been introduced. Their aim is to delineate cell borders using such techniques as: local greyscale thresholding followed by scissoring and morphological thinning [4], [5], scale-space filtering followed by binarization and morphological processing [6] or hexagon detection using shape dependent filters [7], [8], [9]. More sophisticated methods include application of watersheds [10], [11], [12], [13], [14], active contours [15], [16], genetic algorithms [17] or analysis of local pixel levels aimed at finding intensity valleys corresponding to borders between cells [18]. Several machine learning approaches have also been proposed by the team of Ruggeri, including: neural network [19], [20], Bayesian framework [21], support vector machines classifier [22] and genetic algorithm [23]. However, up to this point none of the existing techniques allows to achieve perfect segmentation of endothelial cells. The results

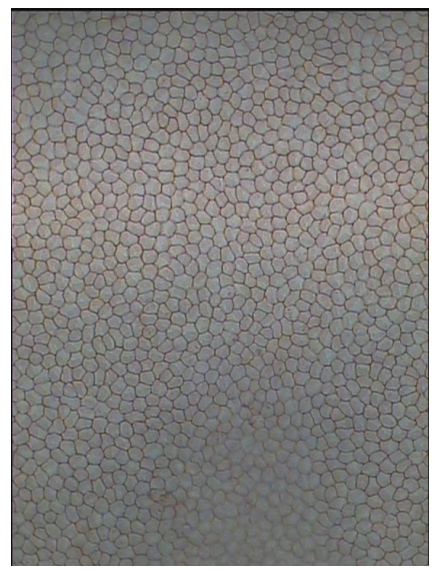

a)

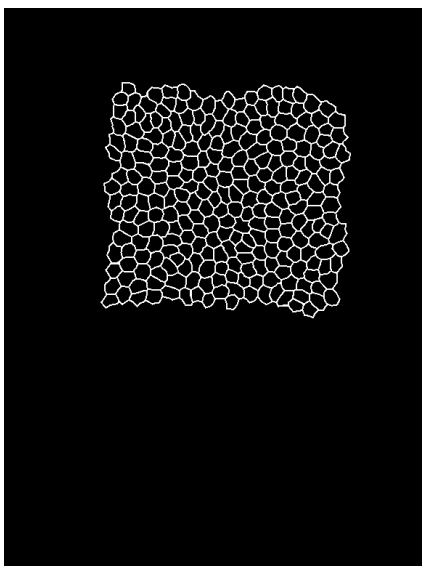

b)
Fig. 1. A sample pair of images from the Alizarine dataset; a) original corneal endothelium image $\mathcal{I}_{R G B}$; b) the corresponding ground truth $\mathcal{G}$. 

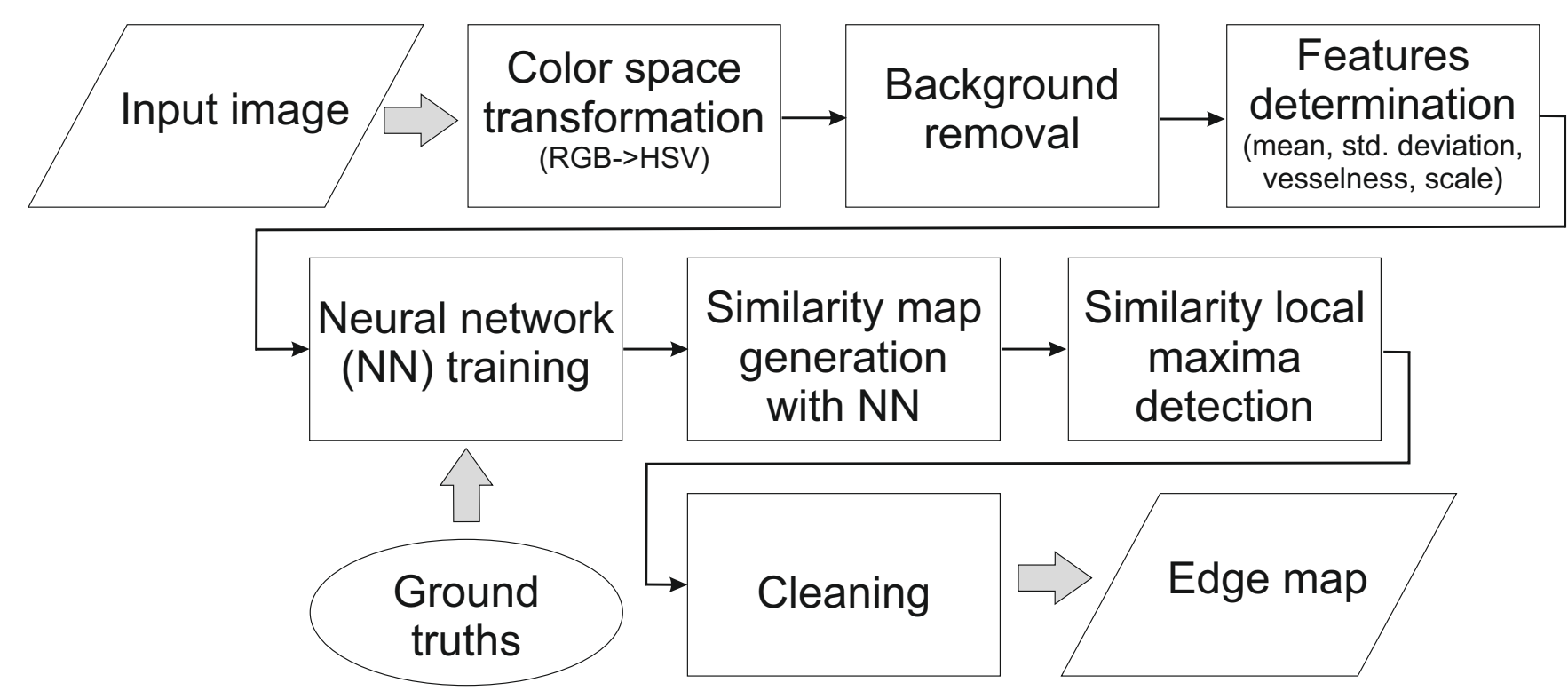

Fig. 2. The general work-flow of the proposed approach for corneal endothelium image segmentation.

still require manual editing, since undetected or false cell boundaries often appear in the resulting image.

Having in mind above limitations, this paper proposes an alternative solution to automatic segmentation of endothelial cells from microscopic images of corneal endothelium. The proposed approach is based on a simple feedforward neural network which is thought to recognize pixels located at the borders between cells and thus segment their contours. The edge probability map output by the network is next subjected to further processing in order to produce one pixel width boundaries of cells. Particularly, local peaks of edge probability map are considered as cell border pixels and linked to create continuous outlines of cells.

The following part of this paper is organised as follows. Firstly, in Section II the description of the dataset used in this study is given. The proposed approach is described in details in Section III and followed by evaluation of the results and discussion in Section IV. Finally, Section V concludes the paper.

\section{INPUT DATA}

In this work corneal endothelium image Alizarine data set was used [20]. The dataset (which can be downloaded from [24]) contains 30 images of corneal endothelium, each stored as JPEG compressed file of the resolution $576 \times 768$ pixels. The images were acquired from 30 porcine eyes stained with alizarine red using inverse phase contrast microscope (CK 40, Olympus) at 200×magnification and analogue camera (SSCDC50AP, Sony).

In the dataset for each image the corresponding manually created ground truth is provided. The ground truth images delineate borders between single cells within selected regions of each image. On average the area of $0.54 \pm 0.07 \mathrm{~mm}^{2}$ per cornea was assessed, ranging from 0.31 to $0.64 \mathrm{~mm}^{2}$.
A sample corneal endothelium image from the considered dataset is shown in Figure 1a, while the corresponding ground truth is presented in Figure 1b. From the figure it can be seen that cells manifest themselves as uniformly sized hexagonal regions separated by visibly darker borders. Due to the acquisition protocol and uneven illumination in some regions of the image the contrast between cell boundaries and background is low. Additionally, intensity inhomogeneity within the background can be observed. These factors significantly hinder cell segmentation.

\section{The Proposed ApProACH}

The aim of the proposed approach is to obtain a binary representation $\mathcal{M}(x, y): \Omega \subset \mathbb{R}^{2} \rightarrow\{0,1\}$ of endothelial cell borders in corneal endothelium image $\mathcal{I}_{R G B}(x, y)$. Particularly, the output of the proposed approach is binary image $\mathcal{M}$ in which 1 (i.e. white pixels) correspond with cell borders and 0 (i.e. black pixels) correspond with cell bodies. This is obtained by following the procedure summarised in Figure 2.

The main idea behind the introduced approach is to use ground truth images provided in Alizarine dataset to train a simple feedforward neural network to recognize borders between cells. The trained network is next used to perform edge based segmentation of endothelial cells in new images. The details of this procedure are given in the following subsections.

\section{A. Colour space transformation and colour component selec- tion}

In the input corneal endothelial images $\mathcal{I}_{R G B}$ each pixel $(x, y)$ stores red, green and blue colour component, i.e. $\mathcal{I}_{R G B}(x, y)=[\mathrm{r}(x, y), \mathrm{g}(x, y), \mathrm{b}(x, y)]$. Prior to the main processing the transformation $\mathrm{F}: \mathcal{I}_{R G B} \rightarrow \mathcal{I}_{H S V}$ into the HSV colour space is applied, where $\mathcal{I}_{H S V}(x, y)=$ 
$[\mathrm{h}(x, y), \mathrm{s}(x, y), \mathrm{v}(x, y)]$ and colour components represent hue, saturation and value respectively. Further processing is performed with respect to $\mathrm{v}$ colour component, since other colour components do not carry significant information related to cell borders. This is illustrated in Figure 3 which presents sample corneal endothelium image and the corresponding $\mathrm{h}, \mathrm{s}, \mathrm{v}$ colour components.

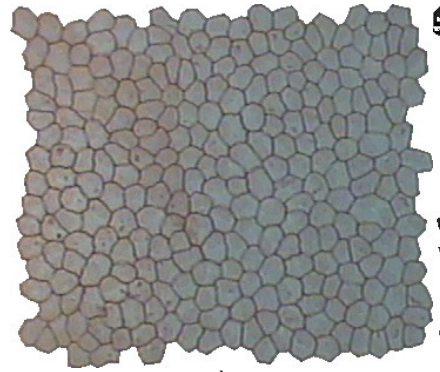

a)

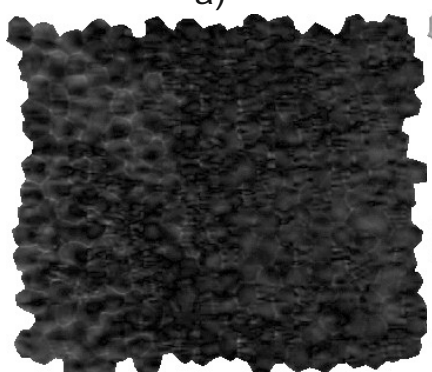

c)

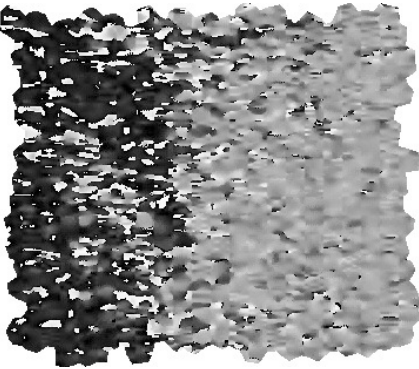

b)

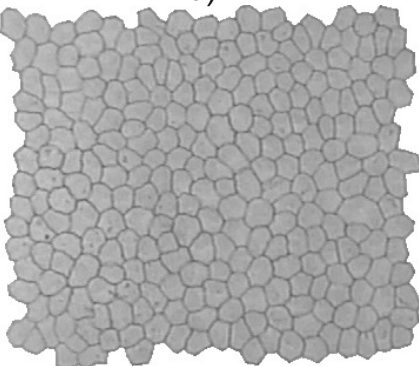

d)
Fig. 3. A corneal endothelium image HSV colour components; a) origina image $\mathcal{I}_{R G B}$; b) hue colour component $\mathrm{h}$; c) saturation colour component $\mathrm{s}$; d) value colour component $\mathrm{v}$.

\section{B. Background removal}

In the next step image $\mathrm{v}$ is enhanced in order to compensate for non-uniform intensity distribution within background and thus to highlight image information at the cell borders. This is obtained via background $\mathrm{v}_{b k g}$ subtraction performed in accordance with the following equation:

$$
\hat{\mathrm{v}}=\mathrm{v}-\mathrm{v}_{b k g}
$$

where image of background is a result of greyscale morphological opening of image $\mathrm{v}$ with a big structural element $\mathrm{s}_{e l}$ (see Eqn. 2).

$$
\mathrm{v}_{b k g}=\left(\mathrm{v} \ominus \mathrm{s}_{e l}\right) \oplus \mathrm{s}_{e l}
$$

where $\ominus$ denotes erosion and $\oplus$ denotes dilation.

The element $\mathrm{s}_{e l}$ should be big enough to remove cell borders. In this study $\mathrm{s}_{e l}$ was selected to be a disk of a radius 15 pixels. Shape of the structural element was set experimentally. Disk shape was used due to similarity to cells shape.

\section{Features determination}

In features determination stage the following features are determined for each pixel $(x, y)$ of an image v:
- average value of intensity $\overline{\mathrm{v}}$ in the neighbourhood of $5 \times 5$ pixels;

- standard deviation of intensity $\sigma_{v}$ in the neighbourhood of $5 \times 5$ pixels;

- vesselness $\mathcal{V}$ determined from image $\mathrm{v}$ using Frangi's approach with default settings [25];

- scale $\mathcal{V}_{\sigma}$ used for vesselness determination [25].

The images representing considered features obtained for a sample image are presented in Figure 4. It can be seen, that vesselness (Fig. 4e), scale (Fig. 4f) and partially standard deviation (Fig. 4d) give visibly distinguished responses at the edges of cells, while intensity information may be helpful in distinguishing cells bodies.

The experiments considering selection of some of the above features were also performed, however using all of these five features yielded the best results in terms of cell detection accuracy.

\section{Neural network training}

Features determined as described above are next composed into a feature vector $\mathrm{F}=\left[\mathrm{v}, \overline{\mathrm{v}}, \sigma_{v}, \mathcal{V}, \mathcal{V}_{\sigma}\right]$ and assigned to the corresponding pixel $(x, y)$. The feature vectors together with the corresponding ground truths segmentations are used to train a neural network $\mathcal{T}$ such that $\mathcal{T}: \mathrm{F}(x, y) \rightarrow\{\mathcal{O}(x, y):$ $\mathcal{O}(x, y) \in[0,1]\}$ and value of 0 corresponds with a cell body while value of 1 denotes a cell boundary.

In the study a simple feedforward neural network of architecture presented in Figure 5 was used. Particularly, the network consists of one hidden layer (with tan-sigmoid transfer function) followed by output layer (with linear transfer function). The hidden layer consists of 10 neurons. Both the number of hidden layers as well as the number of hidden neurons within the layer were adjusted in a trial, balancing between the time required for training and the accuracy of cell borders detection. For training the Levenberg-Marquardt backpropagation approach [26] was used since for the considered problem it provided the best regression between network outputs and network targets. Initial weights and biases were set randomly.

\section{E. Peaks Detection}

The response of a neural network $\mathcal{O}(x, y)$ (i.e. edginess or edge probability map) contains values between 0 and 1 and visibly highlights image information at the borders of cells. However, the response is not everywhere uniform and some weaker boundaries are less highlighted. Additionally, the highlighted edges are few pixels width, thus borders detection can not be accurately performed via image thresholding. Therefore, in order to precisely define border location analysis of local network response maxima is performed via peaks detection. Particularly, the network response image $\mathcal{O}$ is processed independently row by row and column by column. Each row $r_{j}$ such that $\mathcal{O}=\left[r_{j}\right]_{W \times 1}, j \in\{1, \ldots, H\}$ and each column $c_{i}$ such that $\mathcal{O}=\left[c_{i}\right]_{1 \times H}, i \in\{1, \ldots, W\}$ (where $W$, $H$ denote image width and height respectively) is processed separately. The values contained in each column $c_{i}$ and each 


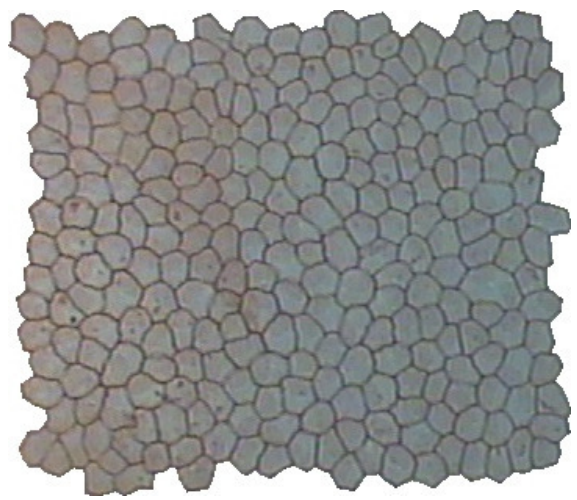

a)

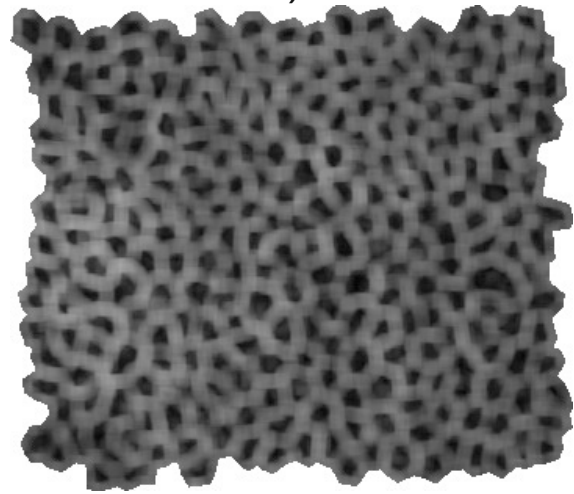

d)

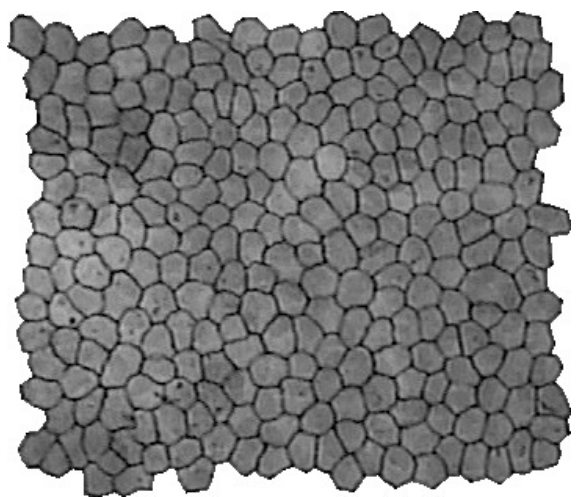

b)

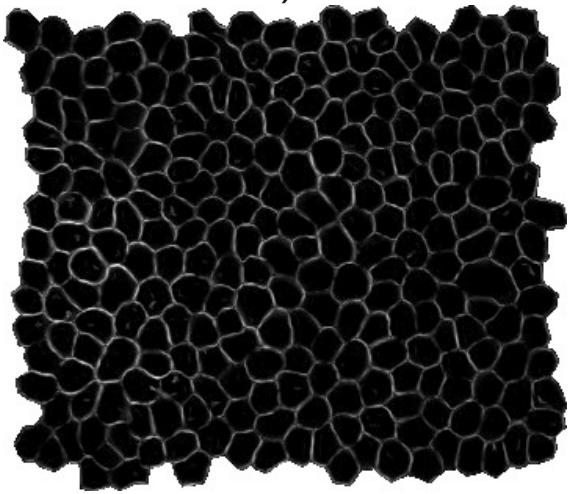

e)

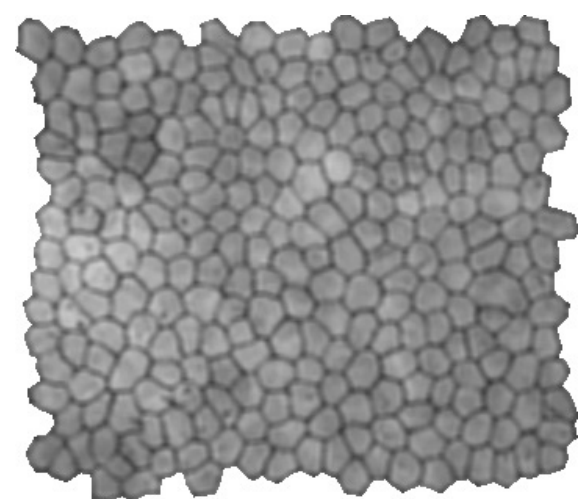

C)

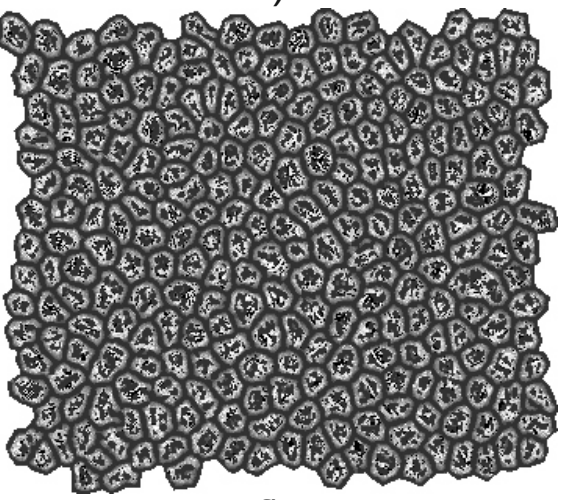

f)

Fig. 4. Image features considered during cells segmentation; a) original colour image $\mathcal{I}_{R G B}$; b) v (value) colour component; c) average $\overline{\mathrm{v}}$; d)standard deviation $\sigma_{v}$; e) vesselness $\mathcal{V}$; f) scale $\mathcal{V}_{\sigma}$.

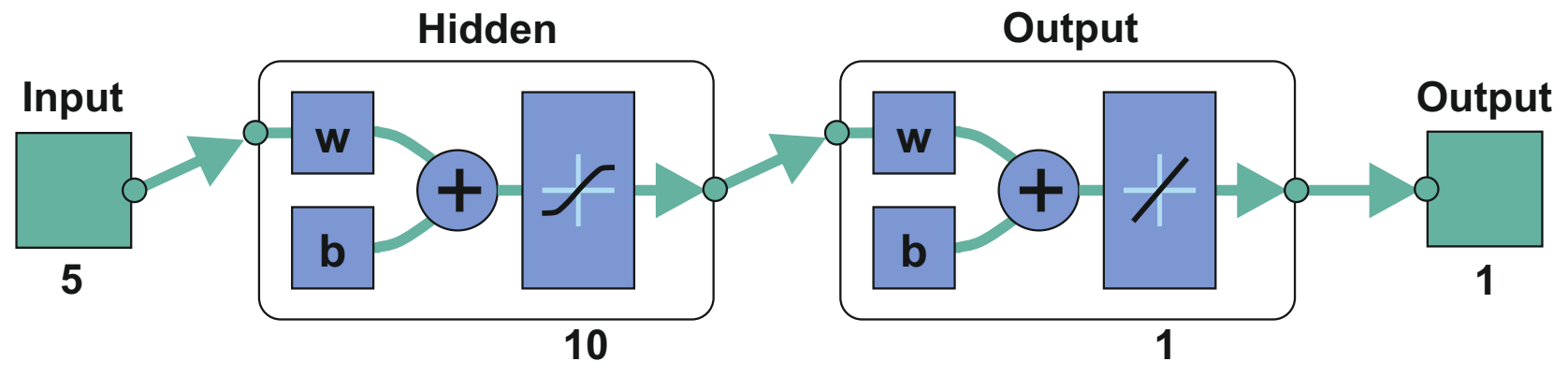

Fig. 5. The structure of a feedforward neural network used in this research.

row $r_{j}$ are treated as a signal in which local peaks indicate edges. A local peak is a data sample that is larger than its nearest neighbouring samples. If a peak is flat, only the point with the lowest index is considered. In order to diminish the number of local maxima around the cell boundaries only peaks higher than some threshold $T_{\text {PeakHeight }}$ are considered. The idea of peaks detection is sketched in Figure 6 which shows the distribution of neural network response over a sample row. Peaks of the accepted height are marked with red circles. The pseudocode of the complete procedure of cell edge candidate detection is shown in Algorithm 1.

\section{F. Cleaning}

Together with real boundary segments, the cell edge candidate detection procedure produces also some small isolated groups of pixels which do not belong to the cell boundaries. In the in the last step of the proposed approach these regions are removed.

The cleaning procedure incorporates a sequence of the following morphological operations performed on a binary image:

- removal of isolated pixels

- dilation with a small structural element

the aim of this step is to close small gaps in the cell boundaries and thus make boundaries continuous; in this 


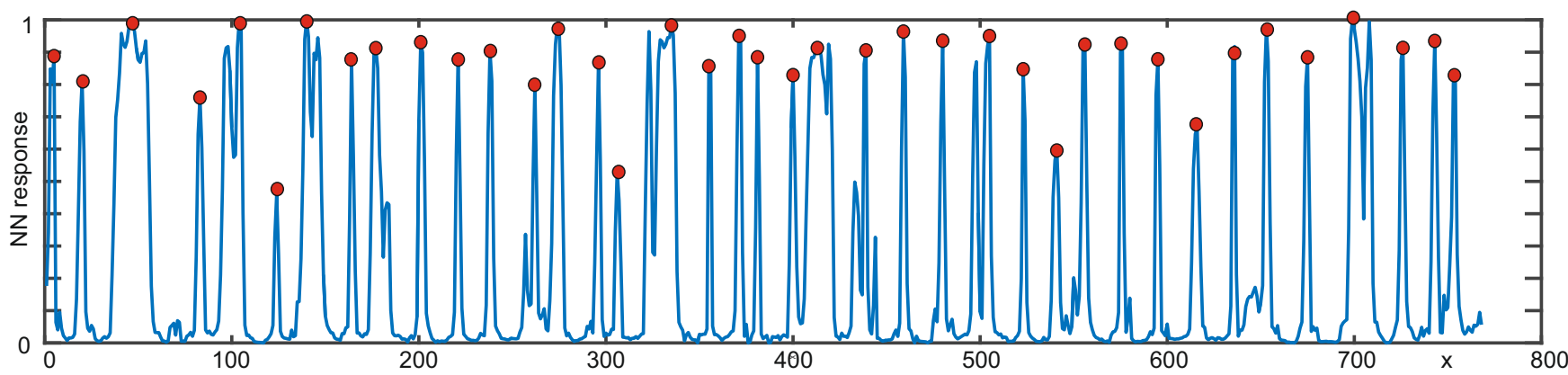

Fig. 6. The idea of local peaks detection. Peaks of the accepted prominence are marked with red circles.

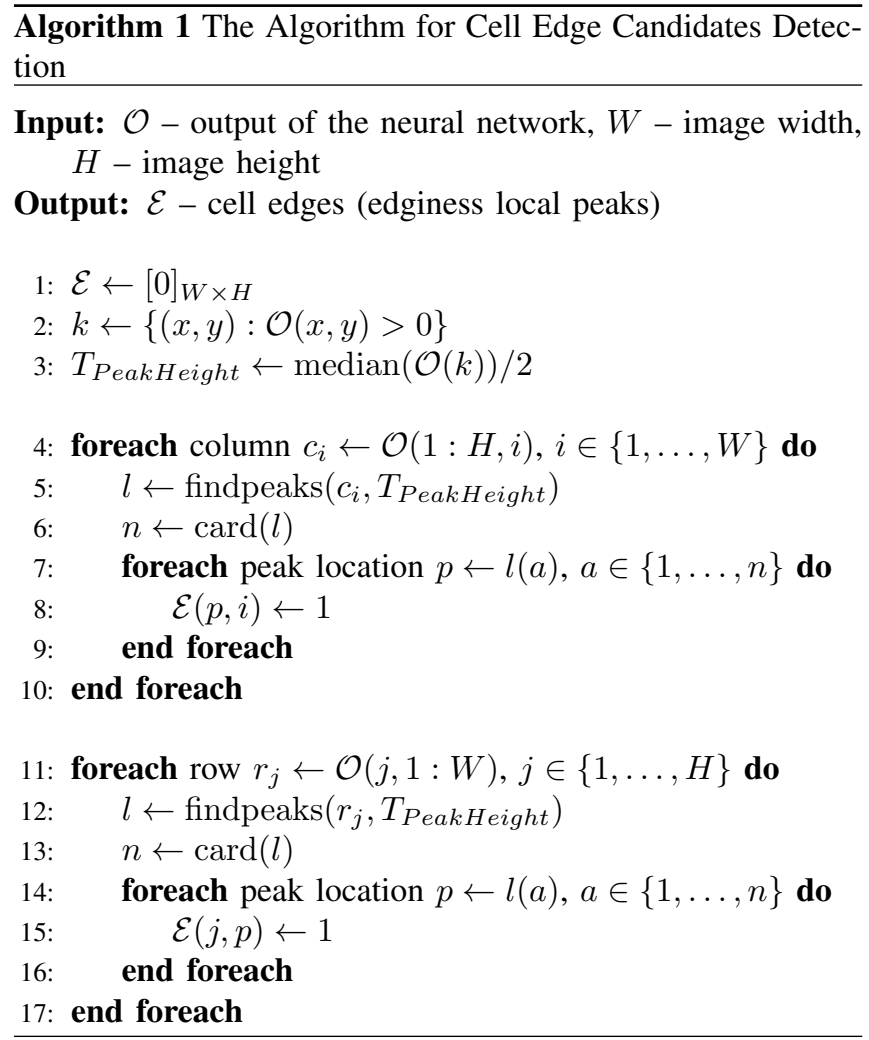

study a square structural element of a size $5 \times 5$ pixels was used;

- skeletonization

for this purpose the iterative thinning is used; the aim of this step is to provide one-pixel-width boundaries of endothelial cells;

- pruning

aiming at removal of spurious branches of skeleton which mostly include fragments of discontinuous boundaries.

The results of the consecutive steps of the proposed approach applied to a sample endothelial image are shown in Figure 7. In particular Figure 7a shows value colour component $v$ of a sample input image. This is followed by image $\hat{v}$ after background removal shown in Figure 7b. The output $\mathcal{O}$ of a feedforward neural network is presented in Figure 7c and followed in Figure 7d by the map of network response local maxima. The map after cleaning $\mathcal{M}$ is presented in Figure $7 \mathrm{e}$ and overlaid on the original image in Figure $7 \mathrm{f}$.

\section{RESUlts AND DisCUSSION}

For verification purposes the endothelial cell Alizarine data set was divided into two equal subsets (i.e. containing 15 images each). First, images denoted by even numbers were used to train the neural network $\mathcal{T}$ (a training set). Next, images denoted by odd numbers were used as a testing set. Particularly, the proposed cell edges detection procedure was applied to each image within the testing set. The network training took about 10 minutes while segmentation of a single image lasted for about 2 seconds (PC computer, 24 GB RAM, Intel Core i7, $3.2 \mathrm{GHz}$ ).

The accuracy of the proposed approach on testing set was assessed twofold. First, the alignment between the ground truths and segmentation results was investigated. Particularly, the results $\mathcal{M}$ of cell segmentation were compared with the ground truth results $\mathcal{G}$ by means of mean square error (MSE), correlation (COR) and DICE measure (DIC) given by Equations 3-5. During the assessment, edge pixels were considered as object. Since in the ground truths $\mathcal{G}$ the edges were few pixels width, they were skeletonized (by thinning) prior to comparison. The results of the above comparison are summarized in Table I with image ID given in the first column and the mean values given in the last row. In the comparison, only the regions with known ground truth borders were considered.

$$
M S E=\frac{1}{K} \sum_{x} \sum_{y}(\mathcal{M}(x, y)-\mathcal{G}(x, y))^{2}
$$

where $K$ denotes a number of pixels in a considered image region.

$$
\begin{gathered}
C O R= \\
\frac{\sum_{x} \sum_{y}(\mathcal{M}(x, y)-\overline{\mathcal{M}})(\mathcal{G}(x, y)-\overline{\mathcal{G}})}{\sqrt{\left(\sum_{x} \sum_{y}(\mathcal{M}(x, y)-\overline{\mathcal{M}})^{2}\right)\left(\sum_{x} \sum_{y}(\mathcal{G}(x, y)-\overline{\mathcal{G}})^{2}\right)}} \\
D I C=\frac{2|\mathcal{M} \cap \mathcal{G}|}{|\mathcal{M}|+|\mathcal{G}|}
\end{gathered}
$$




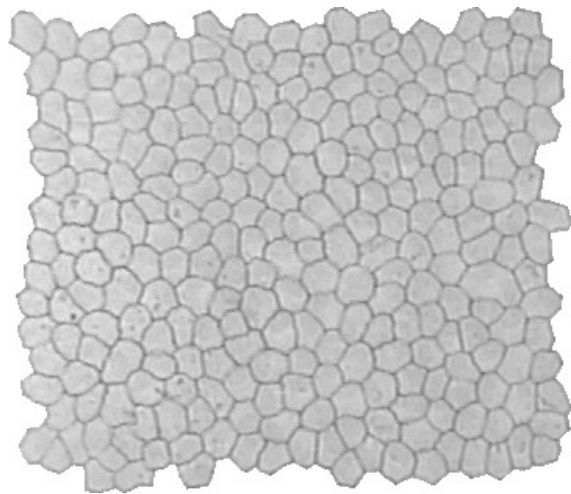

a)

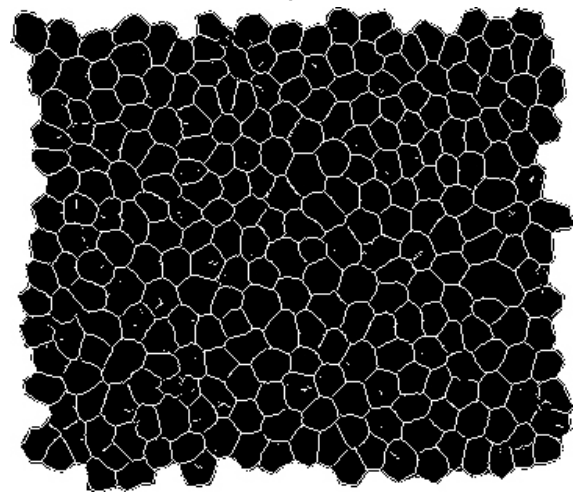

d)

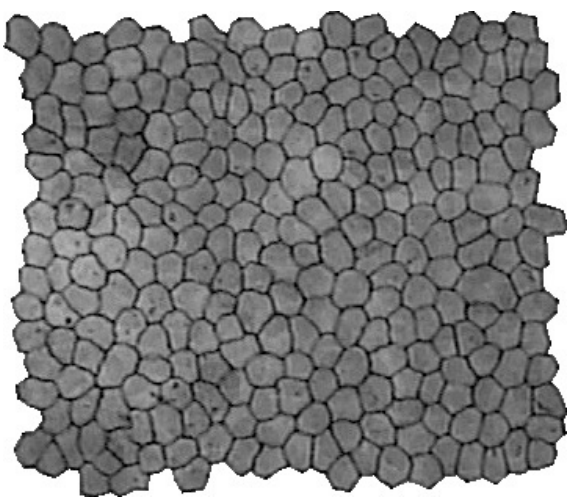

b)

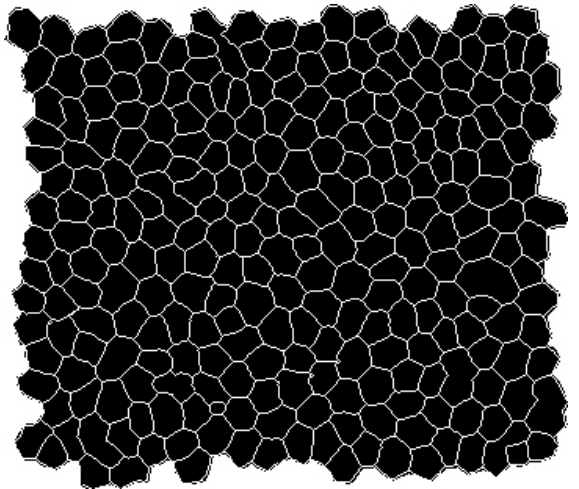

e)

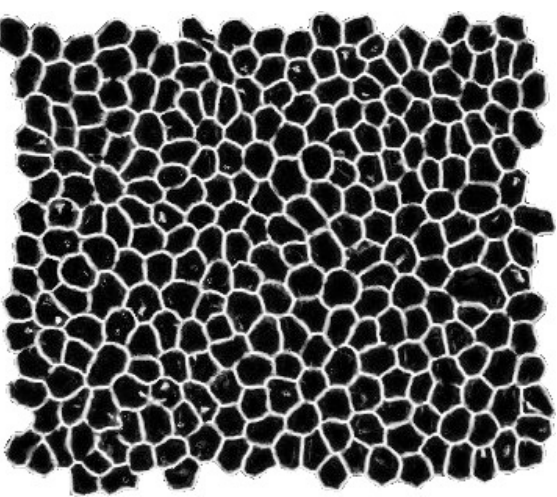

c)

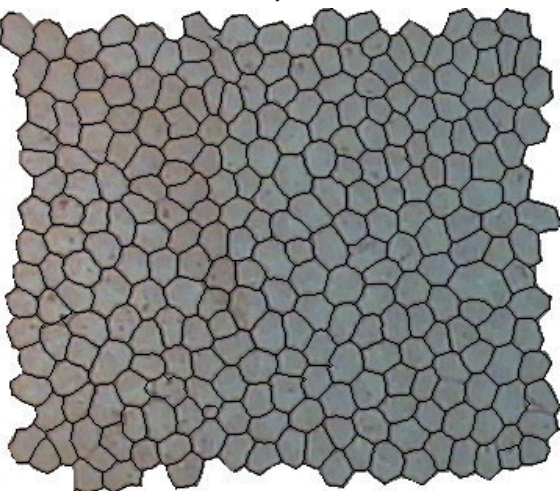

f)

Fig. 7. Consecutive steps of the proposed approach; a) original image $\mathcal{I}_{R G B}$; b) value colour component v; c) output of the neural network $\mathcal{O}$; d) results of local peaks detection; e) final result $\mathcal{M}$ - cleaned and pruned map of local peaks; f) final result overlaid on the original image.

Additionally, the comparison was made between the number $N$ of cells in the segmentation result and in the ground truth image $N_{G T}$ as well as the corresponding average cell sizes $\bar{S}$ and $\bar{S}_{G T}$. Cell sizes were measured in pixels. Additionally, the absolute error $\delta N$ of the determined cell number and the absolute error $\delta \bar{S}$ of the average cell size were calculated according to Equations 6 and 7 respectively.

The results of this comparison were summarised in the Table I with image ID given in the first column and the mean values of errors given in the last row. Again, in the assessment, only the regions with known ground truth borders were considered.

$$
\begin{gathered}
\delta N=\frac{N-n_{G T}}{n_{G T}} \times 100 \% \\
\delta \bar{S}=\frac{\bar{S}-\bar{S}_{G T}}{\bar{S}_{G T}} \times 100 \%
\end{gathered}
$$

The numerical assessment is supplemented by visual results in Figure 8. In the upper panel original images are presented. In the middle pannel the cell edges produced by the proposed approach are overlaid on the original images. Finally, in the bottom panel results are compared with the corresponding ground truths. Particularly, white colour indicates regions where both results overlay. Green colour corresponds to false
TABLE I

THE NUMERICAL ASSESSMENT OF CELL SEGMENTATION ACCURACY WITH RESPECT TO CELL BORDERS ALIGNMENT LEVEL. MSE - MEAN SQUARED ERROR, COR - CORRELATION, DIC - DICE.

\begin{tabular}{|r|c|c|c|}
\hline ID & MSE & COR & DIC \\
\hline 1 & 0.015 & 0.802 & 0.810 \\
\hline 3 & 0.013 & 0.808 & 0.814 \\
\hline 5 & 0.015 & 0.832 & 0.839 \\
\hline 7 & 0.013 & 0.824 & 0.831 \\
\hline 9 & 0.014 & 0.824 & 0.831 \\
\hline 11 & 0.020 & 0.817 & 0.827 \\
\hline 13 & 0.013 & 0.819 & 0.826 \\
\hline 15 & 0.024 & 0.814 & 0.826 \\
\hline 17 & 0.018 & 0.831 & 0.840 \\
\hline 19 & 0.016 & 0.844 & 0.852 \\
\hline 21 & 0.021 & 0.773 & 0.784 \\
\hline 23 & 0.021 & 0.815 & 0.826 \\
\hline 25 & 0.011 & 0.842 & 0.848 \\
\hline 27 & 0.014 & 0.832 & 0.840 \\
\hline 29 & 0.029 & 0.778 & 0.793 \\
\hline \hline avg & 0.017 & 0.817 & 0.826 \\
\hline
\end{tabular}


TABLE II

THE NUMERICAL ASSESSMENT OF CELL SEGMENTATION ACCURACY WITH RESPECT TO MORPHOMETRIC PARAMETERS. $\mathbf{N}$ - THE DETERMINED NUMBER OF CELLS, $\mathbf{N}_{\mathbf{G T}}$ - THE GROUND TRUTH NUMBER OF CELLS, $\delta \mathbf{N}$ - THE ABSOLUTE ERROR OF THE DETERMINED NUMBER OF CELLS, $\overline{\mathbf{S}}$ THE DETERMINED AVERAGE CELL SIZE, $\overline{\mathbf{S}}_{\mathbf{G T}}$ - THE GROUND TRUTH CELL SIZE, $\delta \overline{\mathbf{S}}-$ THE ABSOLUTE ERROR OF THE AVERAGE CELL SIZE.

\begin{tabular}{|r|c|c|c|c|c|c|}
\hline ID & $\mathbf{N}$ & $\mathbf{N}_{\mathbf{G T}}$ & $\delta \mathbf{N}[\%]$ & $\overline{\mathbf{S}}[p x]$ & $\overline{\mathbf{S}}_{\mathbf{G T}}[p x]$ & $\delta \overline{\mathbf{S}}[\%]$ \\
\hline 1 & 264 & 283 & -6.714 & 273,458 & 260.424 & 5.005 \\
\hline 3 & 246 & 264 & -6.818 & 268.984 & 260.280 & 3.344 \\
\hline 5 & 311 & 332 & -6.325 & 292.280 & 275.873 & 5.947 \\
\hline 7 & 258 & 265 & -2.642 & 297.705 & 289.996 & 2.658 \\
\hline 9 & 289 & 303 & -4.620 & 257.114 & 245.845 & 4.584 \\
\hline 11 & 394 & 406 & -2.956 & 300.541 & 292.680 & 2.686 \\
\hline 13 & 237 & 251 & -5.578 & 337.920 & 319.183 & 5.870 \\
\hline 15 & 435 & 467 & -6.852 & 344.414 & 325.949 & 5.665 \\
\hline 17 & 358 & 375 & -4.533 & 343.774 & 328.483 & 4.655 \\
\hline 19 & 359 & 364 & -1.374 & 326.019 & 324.761 & 0.387 \\
\hline 21 & 324 & 356 & -8.989 & 300.213 & 282.986 & 6.088 \\
\hline 23 & 391 & 405 & -3.457 & 331.325 & 321.736 & 2.980 \\
\hline 25 & 248 & 261 & -4.981 & 305.742 & 291.935 & 4.729 \\
\hline 27 & 288 & 300 & -4.000 & 295.892 & 284.637 & 3.954 \\
\hline 29 & 440 & 480 & -8.333 & 347.618 & 318.096 & 9.281 \\
\hline \hline avg & - & - & -5.211 & - & - & 4.522 \\
\hline
\end{tabular}

edges introduced by the proposed approach while the missing edges are shown in magenta. For presentation purposes the best result (case 19, Fig. 8a), the worst result (case 29, Fig. 8b) and the "average" result (case 13, Fig. 8c) were selected.

Based on both the numerical and the visual results it can be concluded, that the proposed approach performs reasonably well. The visual results in Figure 8 clearly show, that the borders produced by the proposed approach and the ground truth are well aligned. This is confirmed by the average values of correlation and DICE equal to 0.817 and 0.826 respectively (see Tab. I). These measures should be considered high, especially having in mind that edges considered here as object are one pixel width and even slight displacement of the edge may decrease these measures, not necessarily meaning that the cell segmentation failed. This effect also can be observed in Figure 8. Additionally, both correlation and DICE measure would have been higher, if the edge pruning hadn't been performed in order to obtain closed borders only. High accuracy of the results is also confirmed with very low MSE on average equal to 0.017 (ranging from 0.013 to 0.029 ).

From Table II it can be seen, that the proposed method slightly underestimates the number $N$ of the detected cells. This in turn increases the average cell size and will increase the determined cell densities. The corresponding, average error $\delta N$ of the determined cell number equals to $-5.2 \%$ (ranging from $-2.6 \%$ to $-9.0 \%$ ) while the resulting cell size determination error is on the average equal to $4.5 \%$ (ranging from $2.6 \%$ to $9.3 \%$ ). In the case of the considered study it corresponded on average to 18 cells which were joined with their neighbours (ranging from 7 cells in the case 19 to 40 cells in the case 29). However, this can be fast corrected by manual editing which in the worst case considered in this study requires drawing c.a. 20 lines and takes definitely less time, than manual segmentation.

\section{CONCLUSIONS}

The proposed approach for endothelial image segmentation provides promising results without user intervention. Additionally, the results are provided in a reasonable time. Although the architecture of a neural network incorporated in the approach was simple, it was capable of delineating accurately most of endothelial cell borders. The results seem even more promising, when one notices that the training dataset contained only several images. It should be also highlighted, that in the ground truth images used for neural network training, only well defined borders were marked. Unsharp and blurry borders were not highlighted and thus it was not possible to train the neural network to recognize this kind of borders. Therefore, the future work will be concentrated on extending the training dataset by corneal endothelium images of low quality in order to make the proposed method capable of segmenting low contrast borders.

\section{ACKNOWLEDGMENT}

This work was co-financed by the Lodz University of Technology, Faculty of Electrical, Electronic, Computer and Control Engineering as a part of a statutory project.

I would also like to acknowledge Dr hab. Adam Piórkowski from AGH University of Science and Technology in Poland for inspiring the research described in this paper.

\section{REFERENCES}

[1] M. Ko, J. Lee, and J. Chi, "Cell density of the corneal endothelium in human fetus by flat preparation," Cornea, vol. 19, no. 1, pp. 80-83, 2000. doi: 10.1097/00003226-200001000-00016

[2] W. Bourne, "Biology of the corneal endothelium in health and disease," Eye, vol. 17, no. 8, pp. 912-918, 2003. doi: 10.1038/sj.eye.6700559

[3] S. Jonuscheit, M. J. Doughty, and K. Ramaesh, "In vivo confocal microscopy of the corneal endothelium: comparison of three morphometry methods after corneal transplantation," Eye, vol. 25, no. 9, pp. 1130âĂŞ1137, 2011. doi: 10.1038/eye.2011.121

[4] G. Ayala, M. Diaz, and L. Martinez-Costa, "Granulometric moments and corneal endothelium status," Pattern Recognition, vol. 34, no. 6, pp. 1219-1227, 2001.

[5] R. Nadachi and K. Nunokawa, "Automated corneal endothelial cell analysis," in Fifth Annual IEEE Symposium on Computer-Based Medical Systems, 1992, pp. 450-457.

[6] F. Sanchez-Marin, "Automatic segmentation of contours of corneal cells," Computers in Biology and Medicine, vol. 29, no. 4, pp. 243 258, 1999.

[7] M. Mahzoun, K. Okazaki, H. Mitsumoto, H. Kawai, Y. Sato, S. Tamura, and K. Kani, "Detection and complement of hexagonal borders in corneal endothelial cell image," Medical Imaging Technology, vol. 14, no. 1, pp. 56-69, 1996.

[8] K. Habrat, M. Habrat, J. Gronkowska-Serafin, and A. Piorkowski, "Cell detection in corneal endothelial images using directional filters," in Image Processing and Communications Challenges 7, ser. Advances in Intelligent Systems and Computing. Springer, 2016, vol. 389, pp. 113 123.

[9] A. Piorkowski, K. Nurzynska, J. Gronkowska-Serafin, B. Selig, C. Boldak, and D. Reska, "Influence of applied corneal endothelium image segmentation techniques on the clinical parameters," Comput. Med. Imag. Grap., vol. 55, pp. 13-27, 2017. doi 10.1016/j.compmedimag.2016.07.010 


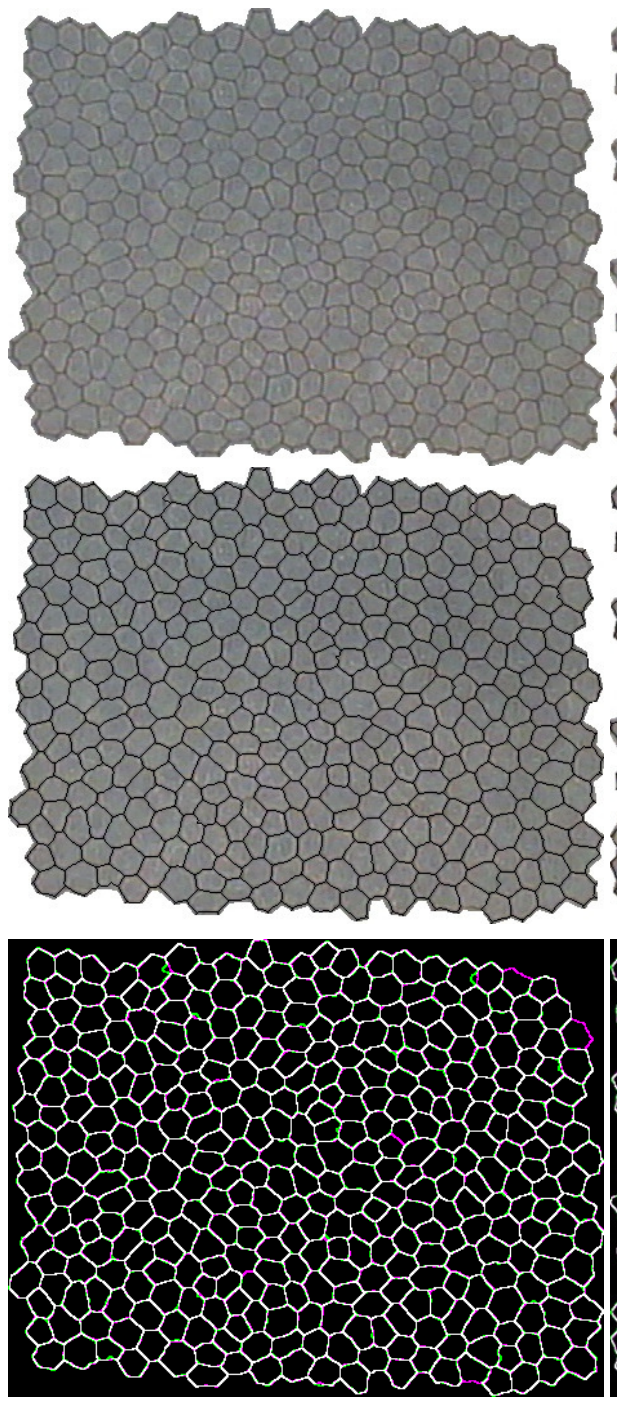

a)
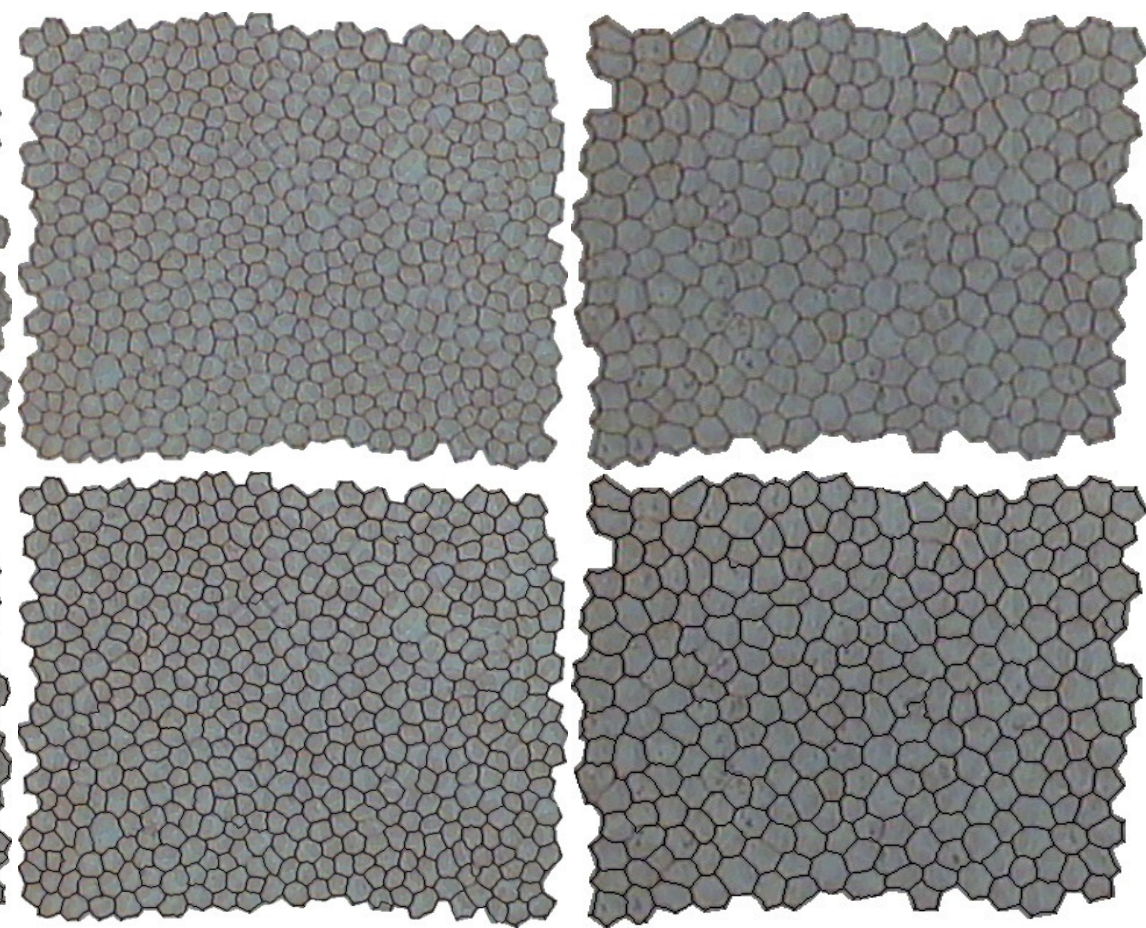

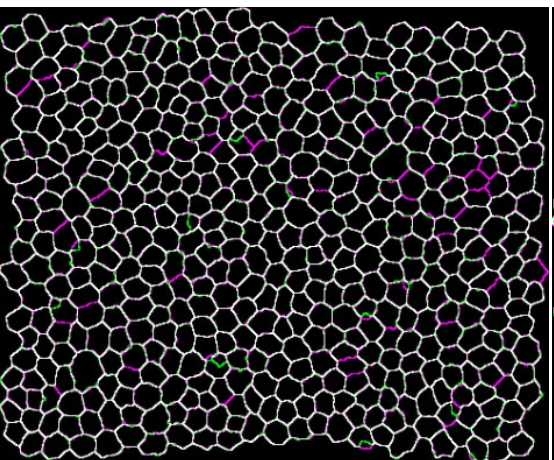

b)

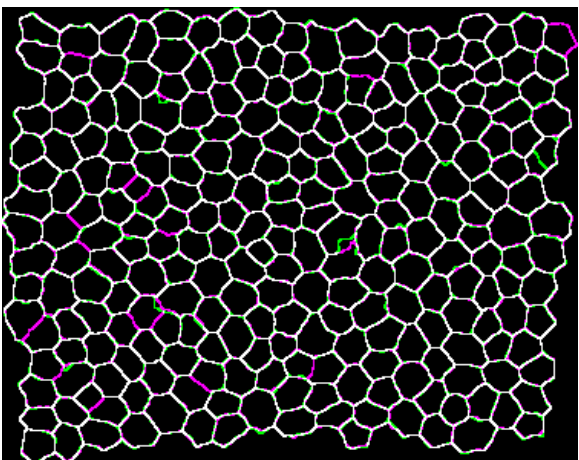

c)

Fig. 8. The results of endothelial image segmentation using the proposed approach; a) the best case; b) the worst case; c) the average case; top panel original images; middle panel - the results overlaid on the original image; bottom panel - comparison with ground truths (magenta - missing edges, green false edges).

[10] L. M. Vincent and B. R. Masters, "Morphological image processing and network analysis of cornea endothelial cell images," pp. 212-226, 1992.

[11] B. Selig, F. Malmberg, and C. L. Luengo Hendriks, "Fast evaluation of the robust stochastic watershed," in Mathematical Morphology and its Applications to Signal and Image Processing : Proceedings of the 12th International Syposium on Mathematical Morphology, Reykjavik, Iceland, ser. Lecture Notes in Computer Science, vol. 9082, no. 9082, 2015, pp. 705-716

[12] J. Angulo and S. Matou, "Automatic quantification of in vitro endothelia cell networks using mathematical morphology," in 5th IASTED International Conference on Visualization, Imaging, and Image Processing (VIIP'05), 2005, pp. 51-56.

[13] Y. Gavet and J.-C. Pinoli, "Visual perception based automatic recognition of cell mosaics in human corneal endothelium microscopy images," Image Analysis \& Stereology, vol. 27, no. 1, pp. 53-61, 2008. doi: 10.5566/ias.v27.p53-61

[14] J. Bullet, T. Gaujoux, V. Borderie, I. Bloch, and L. Laroche, "A reproducible automated segmentation algorithm for corneal epithelium cell images from in vivo laser scanning confocal microscopy," Acta Oph- thalmol., vol. 92, no. 4, pp. e312-e316, 2014. doi: 10.1111/aos.12304

[15] K. Charlampowicz, D. Reska, and C. Boldak, "Automatic segmentation of corneal endothelial cells using active contours," Advances In Computer Science Research, vol. 14, pp. 47-60, 2014.

[16] D. Issam and E. T. Kamal, "Waterballoons: A hybrid watershed balloon snake segmentation," Image Vision Comput., vol. 26, no. 7, pp. 905-912, 2008. doi: 10.1016/j.imavis.2007.10.010

[17] F. Scarpa and A. Ruggeri, "Segmentation of corneal endothelial cells contour by means of a genetic algorithm," in Ophthalmic Medical Image Analysis Second International Workshop, 2015, pp. 25-32.

[18] A. Piorkowski, K. Nurzynska, J. Gronkowska-Serafin, B. Selig, C. Boldak, and D. Reska, "Influence of applied corneal endothelium image segmentation techniques on the clinical parameters," Computer ized Medical Imaging and Graphics, in press.

[19] M. Foracchia and A. Ruggeri, "Cell contour detection in corneal endothelium in-vivo microscopy," in Proceedings of the 22nd Annual International Conference of the IEEE Engineering in Medicine and Biology Society (Cat. No.00CH37143), vol. 2, 2000. doi: 10.1109/IEMBS.2000.897902 pp. 1033-1035 
[20] A. Ruggeri, F. Scarpa, M. De Luca, C. Meltendorf, and J. Schroeter "A system for the automatic estimation of morphometric parameters of corneal endothelium in alizarine red-stained images," British Journal of Ophthalmology, vol. 94, no. 5, pp. 643-647, 2010. doi: 10.1136/bjo.2009.166561

[21] M. Foracchia and A. Ruggeri, "Corneal endothelium cell field analysis by means of interacting bayesian shape models," in 29th Annual Inter national Conference of the IEEE Engineering in Medicine and Biology Society, 2007. doi: 10.1109/IEMBS.2007.4353724 pp. 6035-6038.

[22] E. Poletti and A. Ruggeri, Segmentation of Corneal Endothelial Cells Contour through Classification of Individual Component Signatures. Cham: Springer International Publishing, 2014, pp. 411-414. ISBN 9783-319-00846-2

[23] F. Scarpa and A. Ruggeri., "Development of a reliable automated algorithm for the morphometric analysis of human corneal endothelium," Cornea, vol. 35, no. 9, pp. 1222-1228, 2016. doi 10.1097/ICO.0000000000000908

[24] . Laboratory of Biomedical Imaging and BioImLab, "Endothelial cell Alizarine data set," http://bioimlab.dei.unipd.it/Endo\%20Aliza\% 20Data\%20Set.htm.

[25] A. F. Frangi, W. J. Niessen, K. L. Vincken, and M. A. Viergever, "Multiscale vessel enhancement filtering," ser. Lecture Notes in Computer Science, W. M. Wells, A. Colchester, and S. Delp, Eds., 1998, vol. 1496, pp. 130-137.

[26] D. W. Marquardt, "An algorithm for least-squares estimation of nonlinear parameters," SIAM Journal on Applied Mathematics, vol. 11, no. 2, pp. 431-441, 1963. doi: 10.1137/0111030 